\title{
Fitness compensation and the evolution of selfish cytoplasmic elements
}

\author{
STEPHEN J. FREELAND* \& BRIDGET K. MCCABE \\ Department of Genetics, Downing Street, Cambridge CB2 3EH, U.K.
}

\begin{abstract}
Cytoplasmic incompatibility (CI) and male-killing (MK) are both examples of the action of selfish cytoplasmic elements. Both are found in a wide variety of host arthropod species and sometimes coexist within the same species. Although classical models suggest that fitness compensation is potentially important in explaining the invasion of the MK cytotype, no model of CI evolution incorporates this effect. We construct a discrete-time mathematical model which describes a host population containing both $\mathrm{CI}$ and MK, permitting fitness compensation for survivors of broods which suffer partial mortality as a result of the behaviour of either element. In the absence of fitness compensation, classical models of CI predict the existence of a threshold frequency at which infected individuals must be introduced in order to spread in a wild-type population. We examine whether fitness compensation will affect the ease of spread of CI (reduce the lower threshold) and its equilibrium frequency. Fitness compensation is found to affect the dynamics of $\mathrm{CI}$ in a complex manner. This is so, regardless of whether one considers the introduction of individuals infected with the $\mathrm{CI}$ element alone, or the introduction of individuals infected with both $\mathrm{CI}$ and $\mathrm{MK}$ elements into a wild-type population. The model predicts that fitness compensation has only a small effect on the dynamics of $\mathrm{CI} /$ wild-type population. In contrast, when individuals infected with both $\mathrm{CI}$ and $\mathrm{MK}$ agents are introduced into a wild-type population, the presence of plausible levels of fitness compensation may greatly facilitate the spread of CI. We note that this apparent synergism may be expected from the coexistence of CI with other sex-ratio distorters.
\end{abstract}

Keywords: cytoplasmic incompatibility, fitness compensation, male-killing, mathematical model, Wolbachia.

\section{Introduction}

Selfish cytoplasmic elements (SCEs) are uniparentally inherited endosymbionts which act to maximize their own fitness at the expense of their hosts' fitness (Werren et al., 1988; Hurst, 1993; Hurst et al., 1996). Several classes of such elements have been described. Some act as 'feminizers', causing genetic males to develop and behave as females (see e.g. Rigaud \& Juchault, 1993); others act to transform sexual females into parthenogenetic individuals (see e.g. Stouthamer et al., 1990). In such instances, the benefit to the selfish element is clear. Endosymbionts within infected male hosts effectively have zero fitness, as no vertical transmission may occur. Increasing the number of infected female progeny increases the fitness of the endosymbiont.

'Male-killing' (MK) (Hurst \& Majerus, 1993) and 'cytoplasmic incompatibility' (CI) (Rousset \&

\footnotetext{
${ }^{*}$ Correspondence. E-mail: s.freeland@gen.cam.ac.uk
}

Raymond, 1991) are somewhat more subtle manifestations of selfish behaviour. In the former instance, infected embryos die in the early stages of development if they are male. In the latter instance, sperm from infected males may fertilize infected eggs to produce a viable zygote, but will kill uninfected eggs upon fertilization. The explanations for both types of behaviour rest on the concepts of inclusive fitness and kin selection: in general, Hamilton (1964) has shown that a gene causing an individual to behave to its own detriment will be selectively advantageous if this behaviour benefits other individuals carrying copies of the same gene more than it acts to the detriment of the 'donor'. With regard to MK and CI, the cytoplasmic factors in males have zero fitness so that any action by endosymbionts within males which increases the fitness of infected females will be selectively advantageous regardless of the cost to the male. It is thus hypothesized that the actions of MK represent straightforward kin selection (Skinner, 1985; Hurst, 1991b). Resources which 
would have gone to infected males instead may go to infected females. Alternatively, females may leave fitter progeny as a consequence of a reduction in the rate of inbreeding (Werren, 1987). CI, on the other hand, acts in kin-selective spite (Hurst, 1991a; Rousset \& Raymond, 1991): the bacteria responsible (Wolbachia) act through male sperm to kill progeny of those males which do not possess the same bacteria. The bacteria can increase in frequency simply by reducing the frequency of competitor cytotypes.

From this premise, classical mathematical models of the dynamics of MK incorporate a fitness compensation term (Hurst, 1991b). Such compensation is received by surviving members of a clutch of fertilized eggs which has suffered partial mortality, whether this benefit is realized through a relaxation of sib competition for finite resources and/or through direct cannibalism (Hurst \& Majerus, 1993). For example, recent work has shown that ladybird larvae are more likely to survive to maturity if able to feed on a single dead zygote (Hurst et al., 1997).

Although fitness compensation is recognized as potentially important for the spread of MK within an uninfected host population (Hurst \& Majerus, 1993), no such effect has been considered for CI, despite the fact that many host species exhibit similar ecology or, indeed, are known to host both types of selfish cytoplasmic elements. Nasonia vitripennis, for example, has both $\mathrm{CI}$ induced by Wolbachia (Breeuwer \& Werren, 1990, 1993a, b) and MK caused by a Proteus-like bacterium (Skinner, 1985; Werren et al., 1986). Ladybirds, such as Adalia bipunctata, likewise harbour both Wolbachia (G.D.D. Hurst \& S. West, personal communication) and MK (Hurst et al., 1992; Werren et al., 1994). MK is also quite common within drosophilids (it is best described in the neotropical species; Williamson \& Poulson, 1979); and, given that Wolbachia is also frequently described in drosophilids (Werren et al., 1995), it would seem remarkable if the two did not coexist in some species. In this context, this paper explores the effects of fitness compensation upon the dynamics of $\mathrm{CI}$ with particular reference to three questions.

1 Classical models of CI (see e.g. Fine, 1978; Turelli, 1994) predict the existence of a minimum threshold or proportion of infected individuals necessary for spread of the element through an uninfected population. Does the inclusion of a fitness compensation term lower this threshold value and therefore relax the invasion conditions? If so, one might expect $\mathrm{CI}$, like $\mathrm{MK}$, to be more prevalent in species with reproductive compensation.

2 Some arthropod species apparently exhibit an equilibrium frequency of CI-infected individuals much lower than anything predicted by classical models (Solignac et al., 1994). Does the inclusion of a fitness compensation term help to explain such observations?

3 Suggestion is growing that some species, and perhaps natural populations, host both $\mathrm{MK}$ and $\mathrm{CI}$ (see above). It remains unclear whether, in general, the incidence of coinfection is more common than expected by chance. Were such an association found it might be because the conditions necessary for the spread of MK are also those that aid the spread of CI. Alternatively there may be some population genetical synergism between the two (the spread of one makes the spread of the other easier). A number of possible manifestations of synergism are investigated.

We start by constructing a model for a population which contains both $\mathrm{MK}$ and CI permitting fitness compensation for all survivors within a brood which suffers partial mortality. We then consider the dynamics of $\mathrm{CI}$ alone, the dynamics of $\mathrm{MK}$ alone and finally consider the effect of introducing both selfish elements within the same individuals.

\section{The model}

Consider then a host arthropod species that exists as an infinite panmictic outbred population with discrete generations and with broods produced in isolated batches. For simplicity, females are assumed to mate with only one male prior to production of a brood, and zygotes are produced in a stable 1:1 sex ratio prior to any action by $\mathrm{MK}$.

The host arthropod population comprises four female cytotypes and two male cytotypes: $\mathrm{CI} \sim \mathrm{MK}$ females occurring at frequency $p, \mathrm{CI}$ females occurring at frequency $q$, $\mathrm{MK}$ females occurring at frequency $r$, and wild-type (W) females occurring at frequency $s, \mathrm{CI}$ males occurring at frequency $q_{m}$, and $\mathrm{W}$ males occurring at frequency $s_{m}$ (note, all males inheriting the MK type are assumed to die). Cytotype frequencies for both sexes are always expressed as proportions of the total population of that sex such that $p+q+r+s=1$ and $q_{m}+s_{m}=1$. Both CI and MK agents undergo imperfect vertical transmission during female gametogenesis, at rates $\alpha$ and $\beta$, respectively, (germline cells from a CI-infected female will produce a proportion of eggs infected with $\mathrm{CI}(\alpha)$ and a proportion of wild-type eggs $(1-\alpha)$ ). 
Because of imperfect vertical transmission of both $\mathrm{CI}$ and MK agents, infected cytotype females will produce a mixture of infected and uninfected eggs.

Zygote mortality is determined not only by the presence/absence of the MK type in males, but also by the presence of CI cytotype in parents. Sperm from CI-infected males kill a proportion $(\kappa)$ of eggs not bearing Wolbachia upon fertilization, such that a proportion (1- $\kappa)$ survive. Each type of selfish element confers a fitness cost to its hosts: $U_{\mathrm{ci}}$ and $U_{\mathrm{mk}}$ for $\mathrm{CI}$ and $\mathrm{MK}$, respectively. Multiplicative fitness reduction is assumed for any host infected by both types of selfish element.

Depending upon the mating, a variety of rates of zygote mortality are expected. We assume that those zygotes which do survive will receive a compensatory increase in fitness both from a relaxation of sibling competition for finite resources and from any possible direct utilization of dead zygotes through cannibalism. This increase in fitness is directly related to the proportion of potential siblings killed (the rate of intrabrood mortality), and that the increase in fitness is asymptotic as the proportion of deaths in a brood nears unity (the increase in fitness follows a law of diminishing returns). Two mathematical representations of fitness compensation as a function of mortality which conform to these assumptions are:

$C_{n}=1+\lambda\left(1-e^{-\left(\gamma \chi_{n}\right)^{2}}\right)$ and

$C_{n}=1+\lambda\left(1-e^{-\eta / n}\right)$,

where $\chi$ represents the proportion of zygotes killed within a brood, $\lambda$ and $\gamma$ are constants representing the total amount of compensation available and the proportion of deaths necessary for this maximum to be attained. The first form (1) describes a sigmoidal curve, the second (2) describes an increasing curve whose first derivative is monotonically decreasing.

Investigation of the preceding models under each form of fitness compensation function (eqns 1 and 2) suggests that our results are fairly robust to either choice: no qualitative differences were observed, and quantitative differences were minimal. Unless otherwise stated, results stated within this paper are obtained through the use of the second function (2). Consideration of this function reveals that $1 \leq C_{n} \leq(1+\lambda)$, such that $1+\lambda$ represents an asymptotic maximum of the function.

The proportion of zygotes dying within any given brood $(\chi)$ is dependent on the cytotypes of the two parents. A brood may be produced by any one of eight possible parental matings, each of which yields a different proportion of zygote deaths. These matings and the resulting proportion of deaths expected are given in Table 1.

From the assumptions above (see Table 2) the following recursions may be derived to describe the

Table 1 Proportion of zygote deaths resulting from matings of parents with various cytotypes

\begin{tabular}{cccc}
\hline \multirow{3}{*}{ Parent } & q Parent & $\begin{array}{c}\text { Resulting proportion of } \\
\text { zygote deaths }(\chi)\end{array}$ & $\begin{array}{c}\text { Compensation } \\
\text { term }\left(C_{n}\right)\end{array}$ \\
\hline CI & CI $\sim$ MK & $\frac{\beta+(1-\alpha)(2-\beta) \kappa}{2}$ & $C_{1}$ \\
& CI & $\frac{\beta+\kappa(2-\beta)}{2}$ & $C_{2}$ \\
& MK & $\kappa$ & $C_{3}$ \\
W & W & $\frac{\beta}{2}$ & $C_{4}$ \\
& CI $\sim$ MK & 0 & $C_{5}$ \\
CI & $\frac{\beta}{2}$ & $C_{6}$ \\
MK & 0 & $C_{7}$ \\
W & & $C_{8}$ \\
\hline
\end{tabular}


cytotype frequencies in the following adult generation:

$p^{\prime}=\left[\left(1-U_{\mathrm{ci}}\right)\left(1-U_{\mathrm{mk}}\right)\left(\left(\alpha \beta C_{1} q_{m} p\right)+\left(\alpha \beta C_{5} s_{m} p\right)\right)\right] / \bar{\omega}_{1}$

$q^{\prime}=\left[\left(1-U_{\mathrm{ci}}\right)\left(\begin{array}{c}\left(\alpha(1-\beta) C_{1} q_{m} p\right)+\left(\alpha C_{2} q_{m} q\right) \\ +\left(\alpha(1-\beta) C_{5} s_{m} p\right)+\left(\alpha C_{6} s_{m} q\right)\end{array}\right)\right] / \tilde{\omega}_{1}$

$r^{\prime}=\left[\left(1-U_{\mathrm{mk}}\right)\left(\begin{array}{l}\left((1-\alpha)(1-\kappa) \beta C_{1} q_{m} p\right)+\left(\beta(1-\kappa) C_{3} q_{m} r\right) \\ +\left((1-\alpha) \beta C_{5} s_{m} p\right)+\left(\beta C_{7} S_{m} r\right)\end{array}\right)\right] / \bar{\omega}_{1}$

$s^{\prime}=\left[\begin{array}{l}\left((1-\alpha)(1-\beta)(1-\kappa) C_{1} q_{m} p\right)+\left((1-\alpha)(1-\kappa) C_{2} q_{m} q\right) \\ +\left((1-\beta)(1-\kappa) C_{3} q_{m} r\right)+\left((1-\kappa) C_{4} q_{m} s\right) \\ +\left((1-\alpha)(1-\beta) C_{5} s_{m} p\right)+\left((1-\alpha) C_{6} s_{m} q\right) \\ +\left((1-\beta) C_{7} s_{m} r\right)+\left(s_{m} s\right)\end{array}\right] / \bar{\omega}_{1}$

$q_{m}^{\prime}=\left[\left(1-U_{\mathrm{ci}}\right)\left(\begin{array}{c}\left(\alpha(1-\beta) C_{1} q_{m} p\right)+\left(\alpha C_{2} q_{m} q\right) \\ +\left(\alpha(1-\beta) C_{5} s_{m} p\right)+\left(\alpha C_{6} s_{m} q\right)\end{array}\right)\right] / \bar{\omega}_{2}$

$\left.s_{m}{ }^{\prime}=\left[\begin{array}{l}\left((1-\alpha)(1-\beta)(1-\kappa) C_{1} q_{m} p\right) \\ +\left((1-\alpha)(1-\kappa) C_{2} q_{m} q\right)+\left((1-\beta)(1-\kappa) C_{3} q_{m} r\right) \\ +\left((1-\kappa) C_{4} q_{m} s\right)+\left((1-\alpha)(1-\beta) C_{5} s_{m} p\right) \\ +\left((1-\alpha) C_{6} s_{m} q\right)+\left((1-\beta) C_{7} s_{m} r\right)+\left(s_{m} s\right)\end{array}\right]\right) \bar{\omega}_{2}$,

where $\bar{\omega}_{1}$ is the sum of the numerators of $p^{\prime}, q^{\prime}, r^{\prime}$ and $s^{\prime} ; \bar{\omega}_{2}$ is the sum of the numerators of $q_{m}{ }^{\prime}$ and $s_{m}{ }^{\prime}$.

\section{Dynamics of $\mathrm{Cl}$ with fitness compensation}

When considering a population composed of wildtypes and CI-infected individuals only, the full recursions given above may be simplified by setting $p=r=0$ to produce the following model:

$q^{\prime}=\left[\left(1-U_{\mathrm{ci}}\right)\left(\left(\alpha C_{2} q_{m} q\right)+\left(\alpha C_{6} s_{m} q\right)\right)\right] / \bar{\omega}_{\mathrm{ci}}$

$s^{\prime}=\left[\begin{array}{c}\left((1-\alpha)(1-\kappa) C_{2} q_{m} q\right)+\left((1-\kappa) C_{4} q_{m} s\right) \\ +\left((1-\alpha) C_{6} s_{m} q\right)+\left(s_{m} s\right)\end{array}\right] / \bar{\omega}_{\mathrm{ci}}$

$q_{m}^{\prime}=q^{\prime}$

$s_{m}{ }^{\prime}=s^{\prime}$,

where $\bar{\omega}_{\mathrm{ci}}$ is the sum of the numerators of $q^{\prime}$ and $s^{\prime}$. Equilibria can be found by solving $q^{\prime}=q$, for the condition that $s=1-q$. Three points of equilibrium are found, these being:

(i) $q_{1}^{*}=0, s_{1}^{*}=1$

(ii) $q_{2}^{*}=\frac{-b+\sqrt{\left(b^{2}-4 a c\right)}}{2 a}, s_{2}^{*}=1-q_{2}^{*}$

(iii) $q_{3}^{*}=\frac{-b-\sqrt{\left(b^{2}-4 a c\right)}}{2 a}, s_{3}^{*}=1-q_{3}^{*}$

where

$a=1+C_{2}\left(1-\kappa(1+\alpha)-\alpha U_{\mathrm{ci}}\right)+C_{4}(\kappa-1)+C_{6}\left(\alpha U_{\mathrm{ci}}-1\right)$

$b=C_{6}(1-\alpha)-C_{4}(\kappa-1)-\alpha C_{2}\left(1-U_{\mathrm{ci}}\right)-2$

$c=1-\alpha C_{6}\left(1+U_{\mathrm{ci}}\right)$

The stability of a wild-type population (equilibrium $1 ;(13))$ to invasion by the CI cytotype, when initially the CI type is rare, may be assessed through investigation of the first differential of $q^{\prime}$ at this point. 


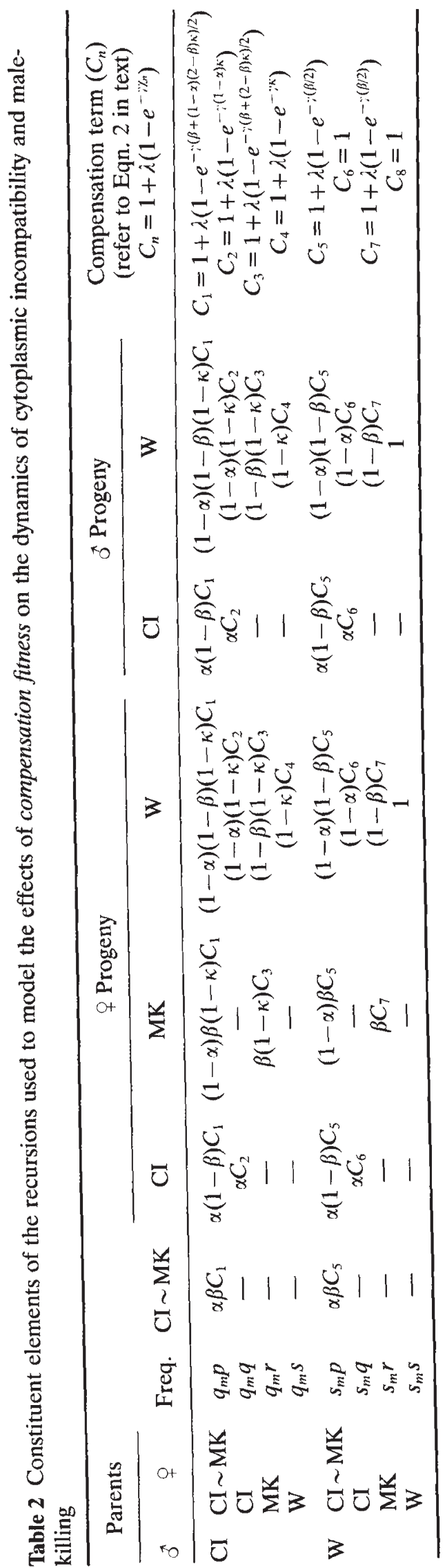

This reveals:

$\left.\frac{\mathrm{d} q^{\prime}}{\mathrm{d} q}\right|_{q=0}=\alpha C_{6}\left(1-U_{\mathrm{ci}}\right)$.

As $C_{6}=1$, it may be observed that

$\left.\frac{\mathrm{d} q^{\prime}}{\mathrm{d} q}\right|_{q=0} \leq 1$

for all plausible values of $\alpha$ and $U_{\text {ci }}$. Therefore, CI will be neutral when appearing in a wild-type population only when it exhibits perfect vertical transmission and is associated with no inherent cost to its host (cf. Fine, 1978). If vertical transmission is less than 100 per cent efficient, or if CI inflicts any fitness cost on its host, then a wild-type population is resistant to invasion.

The lower of the two equilibria $\left(q_{3}^{*}\right)$ represents an unstable point of equilibrium, below which the $\mathrm{CI}$ cytotype will decrease in frequency and above which it will increase in frequency (Fig. 1). In contrast the upper equilibrium $\left(q_{2}^{*}\right)$ is stable (Fig. 1). The CI element therefore must appear above a threshold frequency in order to invade a wild-type host population, whereupon it will increase to a stable upper frequency; this stable upper limit will be at fixation (a)

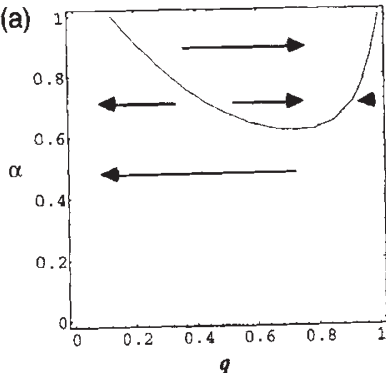

(c)

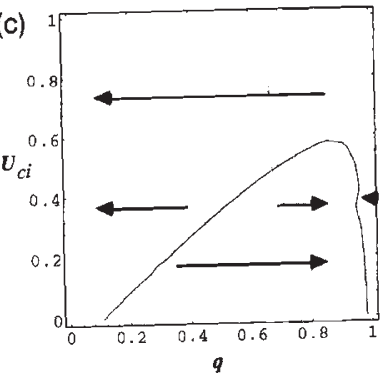

(b)

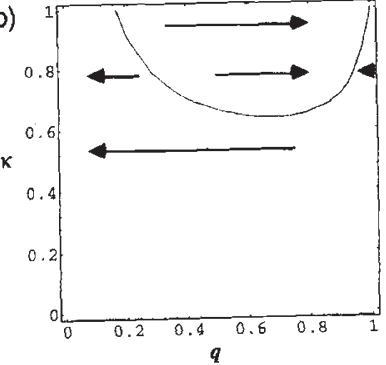

Fig. $1 \mathrm{CI}$ equilibria frequencies $\left(q^{*}\right)$ over a range of values for the following parameters: CI vertical transmission rate $(\alpha)$; CI killing efficiency $(\kappa)$; and cost of $\mathrm{CI}$ element to the host $\left(U_{\mathrm{ci}}\right)$. Unless appearing as a variable, parameters take the following values: $\lambda=1, \gamma=3, \alpha=0.9$, $\kappa=0.9, U_{\mathrm{ci}}=0.1$. 
of CI only when vertical transmission or killing efficiency are perfect, regardless of other parameters (cf. Fine, 1978; Turelli \& Hoffmann, 1995).

Both the lower threshold frequency and the upper stable equilibrium are dependent upon the same parameters: vertical transmission rate $(\alpha)$, killing efficiency $(\kappa)$, fitness cost to host $\left(U_{\mathrm{ci}}\right)$ and fitness compensation from $\mathrm{CI}$-induced death of potential sibs $\left(C_{2}, C_{4}, C_{6}\right)$.

\section{The effect of fitness compensation}

The effect of fitness compensation upon the dynamics of the model is expected to vary qualitatively as well as quantitatively across parameter space. This is understood by considering the extremes of parameter space (Fig. 2): when vertical transmission is perfect $(\alpha=1)$, progeny resulting from a mating involving a $\mathrm{CI}$ female will all be infected with $\mathrm{CI}$, thus no deaths will result and no compensation will occur to CI-bearing individuals. Hence, for high vertical transmission efficiency, compensation cannot aid the spread of CI: any increase in the amount of compensation available may only increase the average fitness of the wild-type cytotype, not only raising the invasion threshold $\left(q_{3}^{*}\right)$ but also lowering the final equilibrium frequency $\left(q_{2}^{*}\right)$ (Fig. 2). In contrast, when killing efficiency is perfect $(\kappa=1)$, the mating between a CI-infected female and a CI-infected male will result in total mortality of the wild-type progeny, thus no uninfected progeny will survive to receive compensation. If any compensation occurs, it will be received by infected individuals because of the death of uninfected sibs (assuming $\alpha<1$ ). This can potentially aid the spread of CI, in that an increase in available compensation raises the average fitness of the CI cytotype only, and leads not only to a reduction in the minimum invasion threshold $\left(q_{3}^{*}\right)$ but also to an increase in the upper stable equilibrium $\left(q_{2}^{*}\right)$ (Fig. 2). When both killing efficiency and vertical transmission are perfect, no compensation will occur in any brood, thus the amount of compensation available is irrelevant to the dynamics of the model.

At any point in parameter space between these extremes (all of the compensation occurs within infected broods vs. all compensation occurs in uninfected broods), compensation affects the fitness of
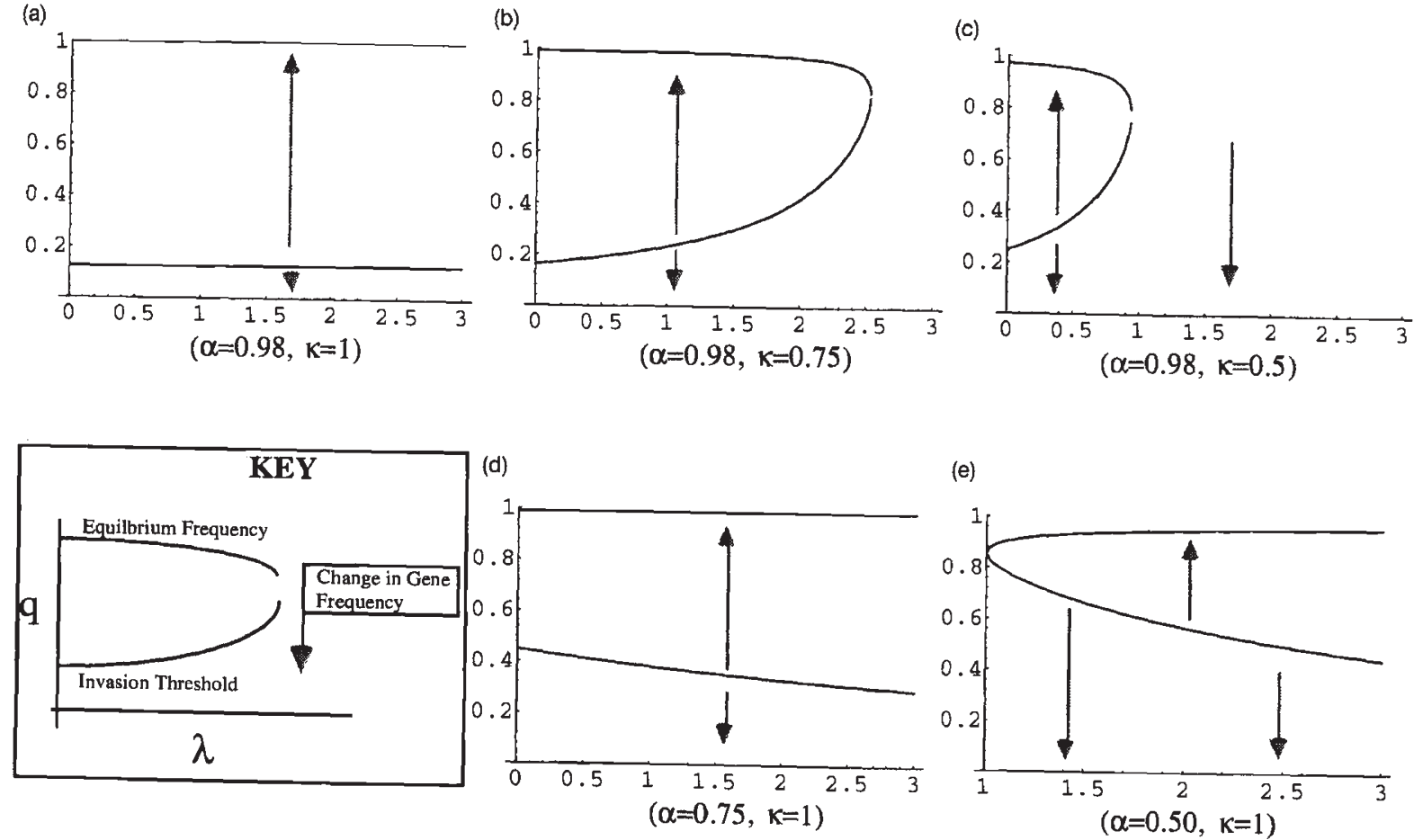

Fig. 2 Changes in invasion thresholds and stable equilibrium frequencies of CI $\left(q_{2}^{*}, q_{3}^{*}\right)$ as a function of fitness compensation $(\lambda)$. Progression through Figs $2 \mathrm{a}, \mathrm{b}$ and $\mathrm{c}$ shows a constant, high vertical transmission rate $(\alpha)$ while the killing efficiency of $\mathrm{CI}(\kappa)$ decreases. Progression through Figs $2 \mathrm{a}$, d and e shows a constant, high killing efficiency $(\kappa)$ while the
vertical transmission rate of CI $(\alpha)$ decreases. 
both $\mathrm{CI}$ and wild-type cytotypes, and it is the relative benefit to each which dictates the effect of varying the amount of available compensation.

Thus, when the rate of vertical transmission is high, fitness compensation may act to reduce the predicted upper equilibrium frequency significantly below anything predicted by classical models. In particular, when vertical transmission rate is very high $(\alpha=0.99)$, cost is insignificant $\left(U_{\mathrm{ci}}=0\right)$ and killing efficiency is low $(\kappa \leq 0.5)$, plausible levels of compensation $(0<\lambda \leq 1)$ may lead to predicted equilibrium frequencies between 55 and 70 per cent (Fig. 3). Furthermore, the value of this upper equilibrium is extremely sensitive to the level of compensation made available. This effect is particularly noticeable when killing efficiency is very low; for example under the stated conditions, when $\kappa=0.06$, an increase in compensation from $\lambda=0$ to $\lambda=0.2$ leads to a decrease of 0.25 in the proportion of $\mathrm{CI}$-infected individuals expected at equilibrium. It is perhaps of interest to note that these parameter values are close to the estimates produced by Solignac et al. (1994) for populations of Drosophila melanogaster. Although the observed rates of 50 per cent or less infected individuals are still lower than anything predicted by the model, we note that fitness compensation may play a role in reducing the stable equilibrium frequency.

We can compare the situation in $D$. melanogaster with that in $D$. simulans. In $D$. simulans, the relevant parameters are: $\kappa \approx 0.3-0.7, U_{\text {ci }} \approx 0-0.05, \alpha \approx 0.97$ (Turelli \& Hoffmann, 1995). Under these parameter values (most notably the higher $\kappa$ ), the effect of

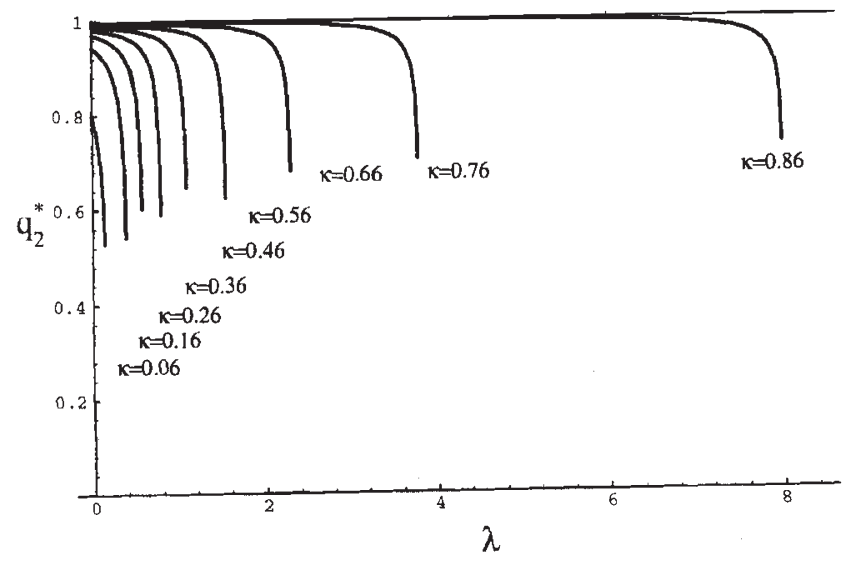

Fig. 3 Variation in the predicted stable upper equilibrium frequency of $\mathrm{CI}\left(q_{2}^{*}\right)$ under different values of killing efficiency $(\kappa)$ and fitness compensation $(\lambda)$, assuming no cost to the host $\left(U_{\mathrm{ci}}=0\right)$.

(c) The Genetical Society of Great Britain, Heredity, 78, 391-402. fitness compensation is negligible, for most realistic values of $\lambda$. The conclusion is that fitness compensation is unlikely to play any significant role in lowering the invasion threshold for $\mathrm{CI}$ entering a wild-type population for more classical forms of strong cytoplasmic incompatibility.

\section{Dynamics of MK with fitness compensation}

When considering a population composed of wildtypes and MK types only, the recursions may be simplified by setting $p=q=q_{m}=0$ to produce the following model:

$r^{\prime}=\left[\left(1-U_{\mathrm{mk}}\right)\left(\beta C_{7} r\right)\right] / \bar{\omega}_{\mathrm{mk}}$

$\left.s^{\prime}=\left[(1-\beta) C_{7} r\right)+s\right] / \bar{\omega}_{\mathrm{mk}}$

$s_{m}{ }^{\prime}=1$,

where $\bar{\omega}_{\mathrm{mk}}$ is the sum of the numerators of $r^{\prime}$ and $s^{\prime}$. This may be considered an extension of a previous model in which the form of compensation was not explicit (Hurst, 1991b). These recursions may be solved to reveal two points of equilibrium which occur at:

$r_{1}^{*}=0, s_{1}^{*}=1$

and

$r_{2}^{*}=\frac{1-\beta C_{7}\left(1-U_{\mathrm{mk}}\right)}{1-C_{7}\left(1-\beta U_{\mathrm{mk}}\right)}, s_{2}^{*}=1-r_{2}^{*}$.

In order to assess the stability of these equilibria, the first differential of the recursion term for $r\left(r^{\prime}\right)$ is taken with respect to $r$ and is then substituted by the terms for each equilibrium. The partial differential of $r^{\prime}$ by $r$ is:

$\frac{\mathrm{d} r^{\prime}}{\mathrm{d} r}=\frac{\beta C_{7}\left(1-U_{\mathrm{mk}}\right)}{\left(r-1-r C_{7}\left(1+\beta U_{\mathrm{mk}}\right)\right)^{2}}$.

The stability of the population to invasion by the MK cytotype can then be found by asking when this is greater than unity at $r=0$. The differential at this value is:

$\left.\frac{\mathrm{d} r^{\prime}}{\mathrm{d} r}\right|_{r=0}=\beta C_{7}\left(1-U_{\mathrm{mk}}\right)$.

Hence, when $\beta \mathrm{C}_{7}\left(1-U_{\mathrm{mk}}\right)>1$ a population at fixation for wild-type is susceptible to invasion by male-killers which will increase in frequency to the equilibrium at $r_{2}^{*}$. The stability of this equilibrium can be investigated by examination of the form of 
$\mathrm{d} r^{\prime} / \mathrm{d} r$ when $r=r_{2}^{*}$. The differential at this value is:

$$
\left.\frac{\mathrm{d} r^{\prime}}{\mathrm{d} r}\right|_{r=\frac{1-\beta C_{7}\left(1-U_{\mathrm{mk}}\right)}{i-C_{7}\left(1-\beta U_{\mathrm{mk}}\right)}}=\frac{1}{\beta C_{7}\left(1-U_{\mathrm{mk}}\right)} .
$$

It may be observed that if invasion is possible (as above in (26)), then this value (27) must always be less than unity, hence this equilibrium is stable.

\section{The effect of fitness compensation}

As noted previously (Hurst, 1991b), a sufficiently large amount of available fitness compensation (represented in eqn 26 as $C_{7}$ ) permits the MK bacterium to invade a wild-type population even when the MK vertical transmission rate is low and/or the inherent cost of MK to the host is high. More importantly, the position of the stable equilibrium frequency can be sensitive to small changes in the vertical transmission rate and, as not previously reported, with the intensity of compensation. It is hence of somewhat dubious value to extrapolate from uncertain measures of the rate of vertical transmission and the degree of compensation to expected equilibrium frequencies.

\section{Dynamics of $\mathrm{MK}$ and $\mathrm{Cl}$ with fitness compensation}

The recursions derived in the Model section describe a host population in which male-killing and cytoplasmic incompatibility coincide. We investigated this through computer simulation, and have chosen to concentrate on the minimum frequency at which the CI-inducing agent must be introduced in order to invade a population (the invasion threshold for $\mathrm{CI}$ ) under two separate scenarios.

First, we consider invasion into a wild-type population, comparing the threshold for $\mathrm{CI}$ appearing as a solitary selfish element (in the form of CI cytotypes) with that of CI appearing alongside MK within dually infected hosts (in the form of $\mathrm{CI} \sim \mathrm{MK}$ cytotypes). Secondly, we consider CI invasion of a population composed of both wild-type and MK individuals at equilibrium frequency (at equilibrium given in the previous section). The introduction into such a population of individuals infected with $\mathrm{CI}$ only is compared with the introduction of individuals infected with both CI and MK.

Individual simulation runs consider the result of introducing a particular starting frequency of either $\mathrm{CI}$ or $\mathrm{CI} \sim \mathrm{MK}$ individuals into the populations described above. A 'result' is assumed to have occurred when all cytotype frequencies remained constant (to six decimal places) between successive generations. The CI agent has invaded if, at equilibrium, the combined frequency of $\mathrm{CI}$ and $\mathrm{CI} \sim \mathrm{MK}$ individuals accounted for 1 per cent or more of the population. Between simulation runs, the starting frequency of $\mathrm{CI} / \mathrm{CI} \sim \mathrm{MK}$ is varied to find the minimum invasion threshold, accurate to six decimal places. When considering the invasion of wild-type/ MK populations at pre-existing equilibria, starting conditions take the introduction of $\mathrm{CI}$ or $\mathrm{CI} \sim \mathrm{MK}$ individuals as migrant additions to the population, rather than as replacements of either cytotype.

The following results refer to simulation runs for varying rates of CI vertical transmission $(\alpha)$, CI killing efficiency $(\kappa)$, and fitness compensation $(\lambda)$, for a fixed rate of MK vertical transmission $(\beta=0.9)$ and fixed cost of both $\mathrm{CI}$ and $\mathrm{MK}$ to their hosts $\left(U_{\mathrm{ci}}=U_{\mathrm{mk}}=0.1\right)$. In the discussion, we use the term 'CI MK threshold' to denote the minimum frequency (invasion threshold) at which the $\mathrm{CI} \sim \mathrm{MK}$ cytotype must first appear in order for CI to invade a population, in the form of either $\mathrm{CI}$ or $\mathrm{CI} \sim \mathrm{MK}$ cytotypes.

\section{Invasion of a wild-type population: comparison of $\mathrm{Cl}$ and $\mathrm{Cl} \sim \mathrm{MK}$ invasion thresholds}

In general, the relationship between the $\mathrm{CI} \sim \mathrm{MK}$ threshold and that of $\mathrm{CI}$ alone is complex, varying both quantitatively and qualitatively over parameter space: in some circumstances the CI $\sim \mathrm{MK}$ threshold is much lower than the CI threshold, in other circumstances, this relationship is reversed. Although this complexity is observed across parameter space as a whole, plausibly high fitness compensation values $(\lambda=0.8)$ can lead to the virtual elimination of the lower CI $\sim \mathrm{MK}$ invasion threshold when the CI vertical transmission rate is high $(\alpha>0.9)$. These results are illustrated in Fig. 4. This is a potentially significant finding as it suggests that uninfected populations invaded by dually infected individuals can easily become populations in which $\mathrm{CI}$ and MK stably coexist, the former at higher frequency, the latter at moderate frequency. The effect may most easily be summarized as CI hitchhiking with MK: compensation causes the spread of the CI $\sim \mathrm{MK}$ cytotype, the subsequent production of $\mathrm{CI}$ cytotypes as failures of $\mathrm{MK}$ vertical transmission and the eventual invasion of $\mathrm{CI} / \mathrm{CI} \sim \mathrm{MK}$ cytotypes.

The diversity of results found through the rest of parameter space seems to result from the interaction of several different effects. CI vertical transmission rate $(\alpha)$ and CI killing efficiency $(\kappa)$ are both directly proportional to the fitness of $\mathrm{CI}$ as a trait, 

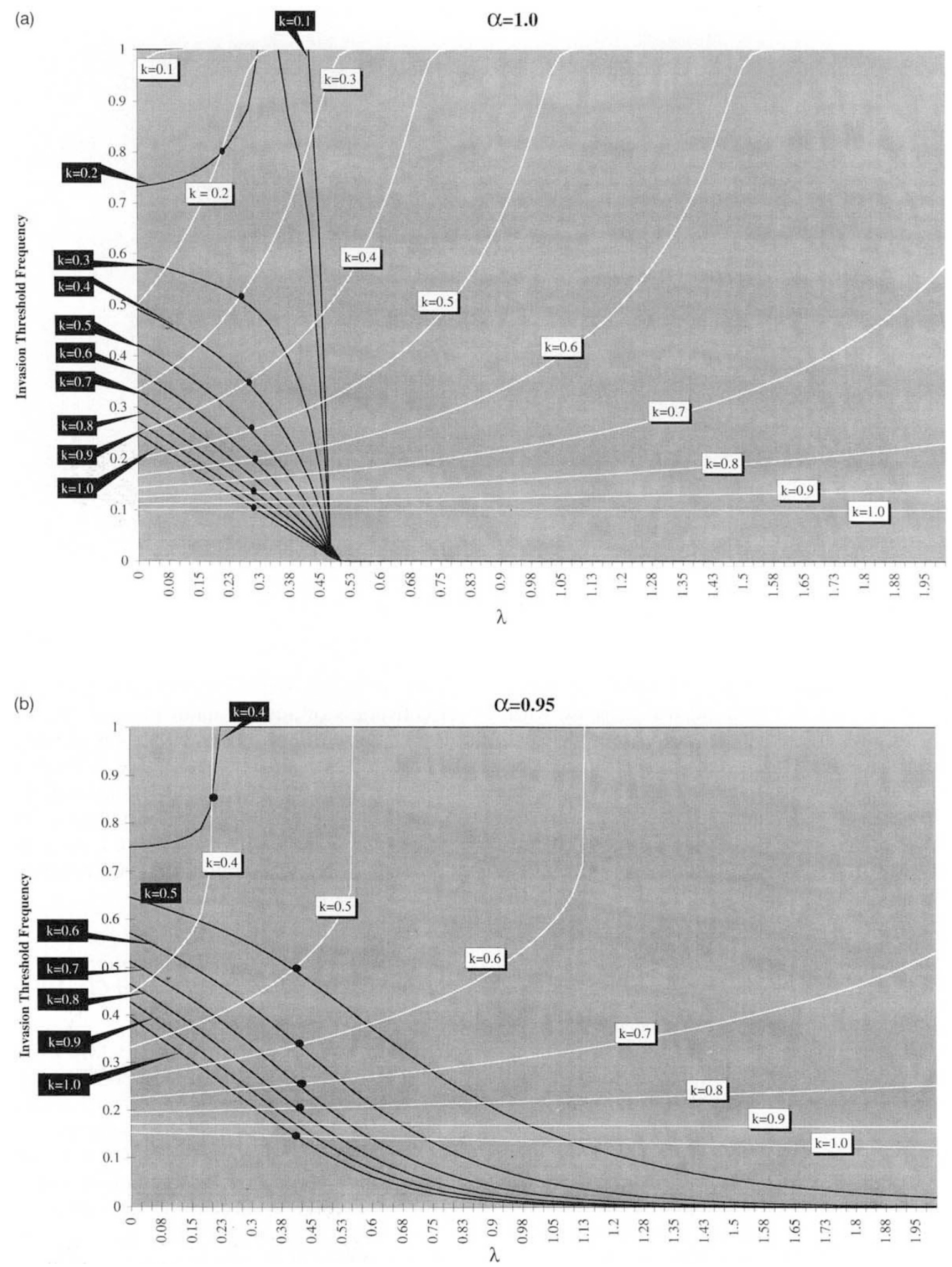

Fig. 4 Cytoplasmic Incompatibility (CI) threshold frequencies (WHITE LINES) and Cytoplasmic Incompatibility with Male-Killing $(\mathrm{CI} \sim \mathrm{MK})$ threshold frequencies (BLACK LINES) for invasion into a wild-type population over a range of compensation values $(\hat{\lambda})$. Threshold frequencies for both cytotypes are shown for a range of values for killing efficiency $(\kappa)$ and vertical transmission rate of $\mathrm{CI}(\alpha)$.

(c) The Genetical Society of Great Britain, Heredity, 78, 391-402. 
(c)

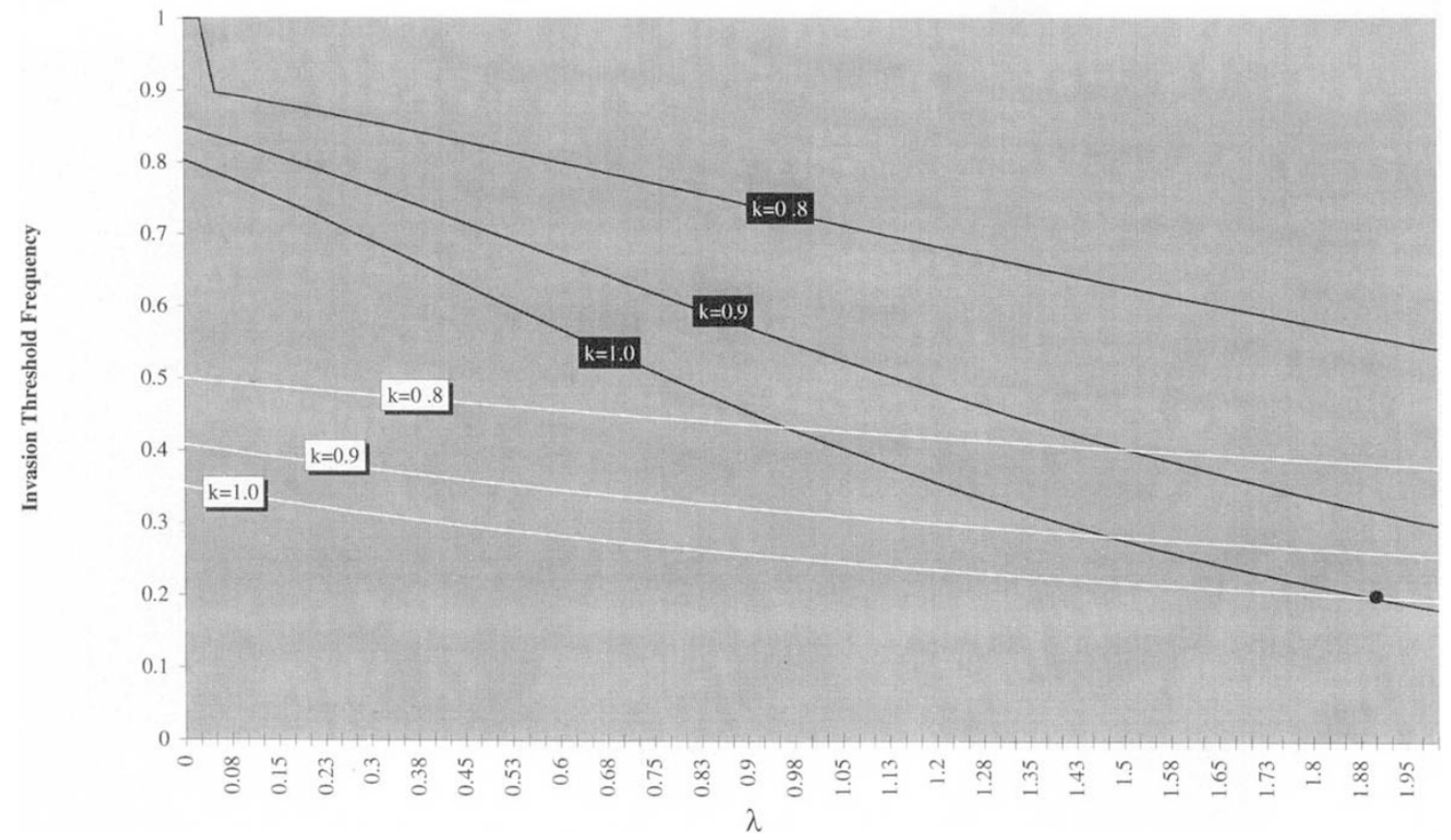

Fig. 4 - cont.

and therefore the fitness of the $\mathrm{CI} \sim \mathrm{MK}$ cytotype. From this, the $\mathrm{CI} \sim \mathrm{MK}$ threshold will increase with decreasing killing efficiency or decreasing vertical transmission frequency for any given level of compensation $(\lambda)$. When compensation is very low, the $\mathrm{CI} \sim \mathrm{MK}$ threshold is higher than the $\mathrm{CI}$ threshold for two reasons: (i) the MK agent needs compensation in order to become a selectively advantageous trait; and (ii) the $\mathrm{CI}$ agent is unable to act when located in the same cytotype as MK (no $\mathrm{CI} \sim \mathrm{MK}$ males survive to sexual maturity). If $\mathrm{CI}$ is to invade a wild-type population, the introduction of $\mathrm{CI} \sim \mathrm{MK}$ individuals must be large enough to produce sufficient $\mathrm{CI}$ males (as failures of MK vertical transmission) to promote the spread of CI. However, without sufficient fitness compensation, the $\mathrm{CI} \sim \mathrm{MK}$-infected individual has the lowest fitness of any cytotype (as a result of multiplicative fitness reduction) and will be lost rapidly from the population. As fitness compensation increases, other effects become important: compensation acts to increase the fitness of $\mathrm{MK}$ as a trait, therefore raising the fitness of the $\mathrm{CI} \sim \mathrm{MK}$ cytotype. Such compensation therefore causes the proportions of $\mathrm{CI} \sim \mathrm{MK}$ and $\mathrm{MK}$ individuals to increase within the first few generations ( $\mathrm{CI}$ hitchhikes with $\mathrm{MK}$ ); this, in turn, produces $\mathrm{CI}$ males at each generation as failures of double vertical transmission and creates a dearth of males within the population, increasing the effectiveness of any CI males present. The combination of these effects ensures that any increase in the amount of compensation available $(\lambda)$ acts to lower the $\mathrm{CI} \sim \mathrm{MK}$ threshold. This relationship is only reversed when CI killing efficiency is very low, significantly reducing the fitness of $\mathrm{CI}$ as a trait and diverting compensation from CI-related deaths towards wild-type individuals.

\section{Invasion of a wild-type/Mk population at equilibrium: comparison of $\mathrm{Cl}$ and $\mathrm{Cl} \sim M K$ invasion thresholds}

Although compensation may lead MK to synergize the invasion by $\mathrm{CI}$ of a wild-type population, no such effect was found when considering CI invasion of a wild-type/MK equilibrium population. This is because the synergism between $\mathrm{CI}$ and $\mathrm{MK}$ noted above relies upon an initial spread of the $\mathrm{CI} \sim \mathrm{MK}$ cytotype: if $\mathrm{MK}$ is already at equilibrium prior to invasion by $\mathrm{CI} \sim \mathrm{MK}$ cytotypes, no such spread will occur and $\mathrm{CI}$ will be unable to invade.

\section{Conclusions}

The model of a CI/wild-type population suggests that fitness compensation plays a complex role in the 
dynamics of CI. When considering the introduction of CI individuals into a population of wild-types, the addition of fitness compensation may act to decrease its predicted invasion threshold and increase its upper equilibrium frequency (when killing efficiency is relatively high and vertical transmission is relatively low) or to increase the invasion threshold and decrease the equilibrium frequency (when killing efficiency is relatively low and vertical transmission frequency is relatively high). Under typical parameter estimates, for example those described for $D$. simulans (Turelli \& Hoffmann, 1995), this model predicts the effect of fitness compensation to be minimal, and we conclude that fitness compensation alone is unlikely to play a major role in explaining the wide phyletic and geographical spread of CI into wild-type populations. In contrast, when vertical transmission efficiency is very high $(\alpha \approx 0.99)$ and killing efficiency is poor $(\kappa \leq 0.5)$, relatively low levels of fitness compensation may act significantly to reduce the stable equilibrium frequency of CI-infected individuals expected. Although this region of parameter space coincides with parameter estimates produced for $D$. melanogaster, our model does not predict the equilibrium frequencies below 50 per cent observed by Solignac et al. (1994).

The full model for a population containing wildtypes, $\mathrm{CI}$ individuals, $\mathrm{MK}$ individuals and $\mathrm{CI} \sim \mathrm{MK}$ individuals suggests that high, yet plausible, levels of fitness compensation may act to cause significant synergism between $\mathrm{CI}$ and $\mathrm{MK}$, such that $\mathrm{CI}$ is able to invade a wild-type population from extremely low starting frequencies if introduced as a dually infected $(\mathrm{CI} \sim \mathrm{MK})$ cytotype. For the most part, this is caused by Wolbachia hitchhiking with MK-inducing bacteria. Thus, we conclude that $\mathrm{CI}$ and MK agents might be expected to coexist in natural populations more often than would be expected from chance alone. We would further expect that species found to host both types of selfish element should exhibit an ecology which permits substantial fitness compensation. Appropriate ecology would include the production of discrete, isolated broods in situations of finite resources; potential brood cannibalism would further enhance compensation availability. We suggest that this effect, beneficial to the spread of $\mathrm{CI}$, might be expected to result from the coexistence of $\mathrm{CI}$ with other sex-ratio distorters such as 'feminizers'.

\section{Acknowledgements}

We would like to thank Laurence Hurst for help with all aspects of the modelling, and John Barrett,
Gilean McVean and Mike Majerus for helpful criticism of the text.

\section{References}

BREEUWER, J. A. J. AND WERREN, J. H. 1990. Microorganisms associated with chromosome destruction and reproductive isolation between two insect species. Nature, 346, 558-560.

BREEUWER, J. A. J. AND WERren, J. H. 1993a. Cytoplasmic incompatibility and bacterial density in Nasonia vitripennis. Genetics, 135, 565-574.

BREEUWER, J. A. J. AND WERREN, J. H. 1993b. Effect of genotype on cytoplasmic incompatibility between two species of Nasonia. Heredity, 70, 428-436.

FINE, P. E. M. 1978. On the dynamics of symbiote-dependent cytoplasmic incompatibility in Culicine mosquitoes. J. Invert. Pathol., 30, 10-18.

HAMILTON, w. 1964. The genetical evolution of social behaviour I and II. J. Theor. Biol., 7, 1-16 \& 17-52.

HURST, G. D. D. AND MAJERUS, M. E. N. 1993. Why do maternally inherited microorganisms kill males? Heredity, 71, 81-95.

HURST, G. D. D., MAJERUS, M. E. N. AND WALKER, L. E. 1992. Cytoplasmic male-killing elements in Adalia bipunctata (Linnaeus) (Coleoptera: Coccinellidae). Heredity, 69, 84-91.

HURST, G. D. D., HURST, L. D. AND MAJERUS, M. E. N. 1997. Cytoplasmic sex ratio distorters. In: O'Neil, S. L., Werren, J. H. and Hoffmann, A. A. (eds) Influential Passengers; Inherited Microorganisms and Invertebrate Reproduction. Oxford University Press, Oxford (in press).

HURST, L. D. 1991a. The evolution of cytoplasmic incompatibility or when spite can be successful. J. Theor. Biol. 148, 269-277.

HURST, L. D. 1991b. The incidences and evolution of cytoplasmic male killers. Proc. R. Soc. Lond. B, 244, 91-99.

HURST, L. D. 1993. The incidences, mechanisms and evolution of cytoplasmic sex ratio distorters in animals. Biol. Rev., 68, 121-193.

HURST, L. D., ATLAN, A. AND BENGTSSON, B. O. 1996. Genetic conflicts. Q. Rev. Biol., 71, 317-364.

Rigaud, T. AND JUChaUlt, P. 1993. Conflict between feminizing sex ratio distorters and an autosomal masculinizing gene in the terrestrial isopod Armadillidium vulgare Latr. Genetics, 133, 247-252.

ROUSSET, F. AND RAYMOND, M. 1991. Cytoplasmic incompatibility in insects: why sterilize females? Trends Ecol. Evol., 6, 54-57.

SKINNER, s. w. 1985. Son-killer: a third extrachromosomal factor affecting sex ratio in the parasitoid wasp Nasonia (= Mormoniella) vitripennis. Genetics, 109, 745-754.

SOLIGNAC, M., VAutrin, D. AND ROUSSET, F. 1994. Widespread occurrence of the proteobacteria Wolbachia and partial cytoplasmic incompatibility in Drosophila melanogaster. C. r. Acad. Sci. Paris Ser. III -Sci. Vie, 317, 461-470. 
STOUTHAMER, R., LUCK, R. F. AND hamilton, w. D. 1990. Antibiotics cause parthenogenetic Trichogramma (Hymenoptera/Trichogrammatidae) to revert to sex. Proc. Natl. Acad. Sci. U.S.A., 87, 2424-2427.

TURELLI, M. 1994. Evolution of incompatibility-inducing microbes and their hosts. Evolution, 48, 1500-1513.

TURELl, M. AND HOFFMANN, A. A. 1995. Cytoplasmic incompatibility in Drosophila simulans: dynamics and parameter estimates from natural populations. Genetics, 140, 1319-1338.

WERREN, J. H. 1987. The coevolution of autosomal and cytoplasmic sex ratio factors. J. Theor. Biol., 124, 317-334.

WERrEn, J. H., NUR, U. AND WU, C.-I. 1988. Selfish genetic elements. Trends Ecol. Evol., 3, 297-302.
WERREN, J. H., SKINNER, S. W. AND HUGER, A. M. 1986. Male-killing bacteria in a parasitic wasp. Science, 231, 990-992.

WERREN, J. H., HURST, G. D. D., ZHANG, W., BREEUWER, J. A. J., STOUTHAmer, R. AND MAJERus, M. E. N. 1994. Rickettsial relative associated with male-killing in the ladybird beetle (Adalia bipunctata). J. Bacteriol., 176, 388-394.

WERREN, J. H., ZHANG, W. AND GUO, L. R 1995. Evolution and phylogeny of Wolbachia: reproductive parasites of arthropods. Proc. R. Soc. Lond. B, 261, 55-63.

Williamson, D. L. AND POUlSon, D. F. 1979. Sex ratio organisms (Spiroplasmas) of Drosophila. In: Whitcomb, R. F. and Tully, J. G. (eds) The Mycoplasmas, pp. 175-208. Academic Press, New York. 